\title{
Avalanche contribution to shear modulus of granular materials
}

\author{
Michio Otsuki ${ }^{1, *}$ and Hisao Hayakawa ${ }^{2}$ \\ ${ }^{1}$ Department of Materials Science, Shimane University, Matsue 690-8504, Japan \\ ${ }^{2}$ Yukawa Institute for Theoretical Physics, Kyoto University, Kyoto 606-8502, Japan
}

(Dated: August 23, 2018)

\begin{abstract}
Shear modulus of frictionless granular materials near the jamming transition under oscillatory shear is numerically investigated. It is found that the shear modulus $G$ satisfies a scaling law to interpolate between $G \sim\left(\phi-\phi_{J}\right)^{1 / 2}$ and $G \sim \gamma_{0}^{-1 / 2}\left(\phi-\phi_{J}\right)$ for a linear spring model of the elastic interaction between contacting grains, where $\phi, \phi_{J}$, and $\gamma_{0}$ are, respectively, the volume fraction of grains, the fraction at the jamming point, and the amplitude of the oscillatory shear. The linear relation between the shear modulus and $\phi-\phi_{J}$ can be understood by slip avalanches.
\end{abstract}

PACS numbers: 83.80.Fg, 81.40.Jj, 62.20.fq

\section{INTRODUCTION}

Amorphous materials consisting of densely packed particles such as granular materials [1], colloidal suspensions [2], emulsions, and foams [3] have rigidity above a critical density, while they lose rigidity below the critical density. Such rigidity transition, known as the jamming transition, has attracted much attention among researchers in these days [4].

In the vicinity of the jamming point taking place at the volume fraction of the jamming point $\phi_{J}$, amorphous materials exhibit critical behavior. Assemblies of frictionless particles exhibit a mixed transition, in which the coordination number shows a discontinuous transition, while the pressure, the elastic moduli, and the characteristic frequency of the density of state exhibit continuous transition [5 7]. Moreover, critical scaling laws, similar to those observed in equilibrium critical phenomena, exist in the rheology of the sheared disordered particles 8 22]. On the other hand, assemblies of soft frictional grains exhibit a discontinuous transition associated with a hysteresis loop and a discontinuous shear-thickening in the rheology under steady shear [23 31].

The shear modulus $G$, the ratio of the shear stress to the shear strain, is one of the most important quantities to characterize the jamming transition. It is well known that $G$ slightly above the jamming point satisfies the scaling

$$
G \sim\left(\phi-\phi_{J}\right)^{1 / 2}
$$

for grains interacting by a linear spring model, where $\phi$ is the volume fraction [5 7]. This power law as well as the frequency dependence of $G$ can be explained by the analysis of the soft mode, and the validity of these laws are verified through simulations [32, 33]. On the other hand, Refs. 34, 35] have recently reported that $G$ might obey a different power law of the excess volume fraction,

*otsuki@riko.shimane-u.ac.jp
$\phi-\phi_{J}$, as

$$
G \sim \gamma_{0}^{-c}\left(\phi-\phi_{J}\right)
$$

through an experiment and a simulation of soft spherical particles at finite temperature though the strain amplitude $\gamma_{0}$ dependence with an exponent $c$ has not been discussed. The conflict between Eqs. (1) and (2) may be understood from the amplitude of the shear strain. Indeed, conventional studies assume that the contact network is unchanged during the process because of an infinitesimal amplitude of the shear strain, but might be inappropriate for a finite strain even near the jamming point. In fact, as shown in Fig. 1 obtained from a simulation under an oscillatory shear, many bonds between contacting grains near the jamming point are broken under the shear strain $\gamma$ larger than $10^{-4}$, which causes slip avalanches distributed in a broad range of sizes [36 38]. To interpolate previously reported relations, Eqs. (11) and (2), we postulate the scaling for the shear modulus:

$$
G\left(\phi, \gamma_{0}\right)=G_{0}\left(\phi-\phi_{J}\right)^{a} \mathcal{G}\left(\gamma_{0} /\left(\phi-\phi_{J}\right)^{b}\right),
$$

where $a$ and $b$ are the critical exponents, and $G_{0}$ is the characteristic shear modulus, which is determined from the elasticity and the diameter of grains. We also assume that the scaling function $\mathcal{G}(x)$ satisfies

$$
\lim _{x \rightarrow 0} \mathcal{G}(x)=\text { const., } \quad \lim _{x \rightarrow \infty} \mathcal{G}(x)=x^{-c} .
$$

To be consistent with the known results, the exponents should satisfy $a=1 / 2$ and $a+b c=1$. A similar analysis on the non-linear rheology of an unchanged contact network is reported in Ref. [39], but they do not discuss the effect of the slip avalanches. It should be noted that the plastic-elastic rheology of jammed granular materials under large strain amplitude is studied in Ref. [40], but the studies of the shear modulus depending on the stress avalanche by the shear strain do not exist as long as we know.

In this paper, we numerically study the behavior of the shear modulus $G$ of granular materials near the jamming point $\phi_{J}$ under an oscillatory shear. In Sec. II we explain our setup and model. In Sec. [II] we present the details of 
(a)

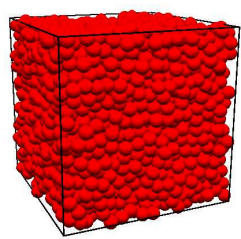

(c)

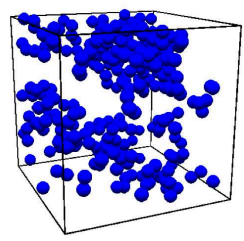

(b)

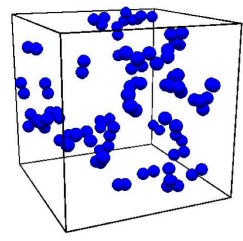

(d)

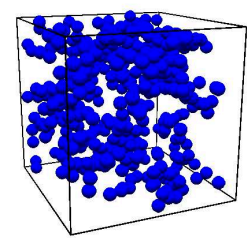

FIG. 1: (Color online) Time evolution of a frictionless granular system of a linear spring between contacting grains for the packing fraction $\phi=0.660$ under an oscillatory shear, where the jamming fraction $\phi_{J}$ is 0.6494 . (a) A snapshot of grains without shear strain, i.e. $\gamma=0$. (b) A snapshot of the grains whose bonds between contacting pairs at $\gamma=0$ are broken at $\gamma=1.2 \times 10^{-4}$. (c) A snapshot of the grains whose bonds between contacting pairs at $\gamma=0$ are broken at $\gamma=4.8 \times 10^{-4}$. (d) A snapshot of the grains whose bonds between contacting pairs at $\gamma=0$ are broken at $\gamma=7.5 \times 10^{-4}$.

our numerical results. In Sec. IV] we phenomenologically estimate the values of the exponents $a, b$, and $c$ we have introduced. We determine the values of exponent $a$ in Eq. (3) in terms of a phenomenological argument in Sec. IV A estimate the exponent $c$ in the asymptotic form (4) caused from the slip avalanches in Sec. IVB and discuss the exponent $b$ in Eq. (3) in Sec. IVC In Sec. V] we discuss and conclude our results. In Appendix $\mathbf{A}$, we explain the method to determine the jamming transition point. In Appendix B] we re-derive the size distribution of the avalanche obtained in Ref. [36].

\section{SETUP OF OUR SIMULATION}

Let us consider a three-dimensional frictionless granular assembly in a cubic box of the linear size $L$. The system includes $N$ spherical grains, where each of them has an identical mass $m$. The position and the velocity of the grain $i$ are, respectively, denoted by $\boldsymbol{r}_{i}$ and $\boldsymbol{v}_{i}$. There exist 4 types of grains for diameter, $0.7 d_{0}$, $0.8 d_{0}, 0.9 d_{0}$, and $d_{0}$, where number of each species is $N / 4$. Throughout this paper, we use the volume fraction $\phi$ to characterize the density of the grains.

Because the grains are frictionless, the contact force has only the normal component of the elastic force $\boldsymbol{f}_{i j}^{(\mathrm{el})}$ and the dissipative force $\boldsymbol{f}_{i j}^{(\mathrm{dis})}$, which are respectively

given by

$$
\begin{aligned}
\boldsymbol{f}_{i j}^{(\mathrm{el})} & =k\left(d_{i j}-r_{i j}\right)^{\Delta} \Theta\left(d_{i j}-r_{i j}\right) \boldsymbol{n}_{i j}, \\
\boldsymbol{f}_{i j}^{(\mathrm{dis})} & =-\eta v_{i j} \Theta\left(d_{i j}-r_{i j}\right) \boldsymbol{n}_{i j}
\end{aligned}
$$

with the elastic constant $k$, the viscous constant $\eta$, the diameter $d_{i}$ of grain $i, \boldsymbol{r}_{i j} \equiv \boldsymbol{r}_{i}-\boldsymbol{r}_{j}, \boldsymbol{n}_{i j} \equiv \boldsymbol{r}_{i j} / r_{i j}, r_{i j} \equiv$ $\left|\boldsymbol{r}_{i j}\right|, d_{i j} \equiv\left(d_{i}+d_{j}\right) / 2$, and $v_{i j} \equiv\left(\boldsymbol{v}_{i}-\boldsymbol{v}_{j}\right) \cdot \boldsymbol{n}_{i j}$. Here, $\Theta(x)$ is the Heaviside step function satisfying $\Theta(x)=1$ for $x \geq 0$ and $\Theta(x)=0$ for otherwise. The exponent $\Delta$ characterizes the elastic repulsive interaction, i.e. $\Delta=$ $3 / 2$ for spheres of Hertzian contact force, and $\Delta=1$ for the linear spring model. Note that the characteristic shear modulus $G_{0}$ introduced in Eq. (3) corresponds to $k d_{0}^{\Delta-2}$.

In this paper, we apply an oscillatory shear along the $y$ direction under the Lees-Edwards boundary condition [41]. As a result, there exists macroscopic displacement only along the $x$ direction. The time evolution of such a system, known as the SLLOD system [41], is given by

$$
\begin{aligned}
\frac{d \boldsymbol{r}_{i}}{d t} & =\frac{\boldsymbol{p}_{i}}{m}+\dot{\gamma}(t) y_{i} \boldsymbol{e}_{x}, \\
\frac{d \boldsymbol{p}_{i}}{d t} & =\sum_{j \neq i}\left\{\boldsymbol{f}_{i j}^{(\mathrm{el})}+\boldsymbol{f}_{i j}^{(\mathrm{dis})}\right\}-\dot{\gamma}(t) p_{i, y} \boldsymbol{e}_{x}
\end{aligned}
$$

where $\boldsymbol{p}_{i}$ and $\boldsymbol{e}_{x}$ are respectively the peculiar momentum and the unit vector parallel to the $x$ direction.

We use the viscous constant $\eta=1.0 \sqrt{m k d_{0}^{\Delta-1}}$, which corresponds to the constant restitution coefficient $e=$ 0.043 for $\Delta=1$. We adopt the leapfrog algorithm, the second-order accuracy in time with the time interval $\Delta t=0.2 \tau$, where $\tau$ is the characteristic time of the stiffness, i.e. $\tau=\sqrt{m d_{0}^{1-\Delta} / k}$. The number $N$ of the particles is 16000 except in Appendix $\mathrm{A}$ where we estimate the jamming point $\phi_{J}$ from a finite size scaling. We have verified that the shear modulus is almost independent of the system size for $N \geq 4000$.

We randomly place the grains in the system as an initial state, and wait until the kinetic energy of each grain becomes smaller than $10^{-14} k d_{0}^{1+\Delta}$. Then, we apply the shear with the shear rate

$$
\dot{\gamma}(t)=\gamma_{0} \omega \sin (\omega t),
$$

where time $t$ is measured from the relaxed static configuration and $\omega$ is the angular frequency of the oscillatory shear. From Eq. (9), the shear strain is given by

$$
\gamma(t)=\gamma_{0}\{1-\cos (\omega t)\} .
$$

We examine the shear modulus for various strain amplitudes $\gamma_{0}=10^{0}, 10^{-1}, 10^{-2}, 10^{-3}, 10^{-4}$, and $10^{-5}$ for $\omega \tau=10^{-4}$ [42]. We analyze the real part of the complex shear modulus [43] (storage modulus) defined by

$$
G\left(\phi, \gamma_{0}, \omega\right)=-\frac{\omega}{\pi} \int_{t_{0}\left(\gamma_{0}\right)}^{2 \pi / \omega+t_{0}\left(\gamma_{0}\right)} d t \frac{S(t) \cos (\omega t)}{\gamma_{0}},
$$




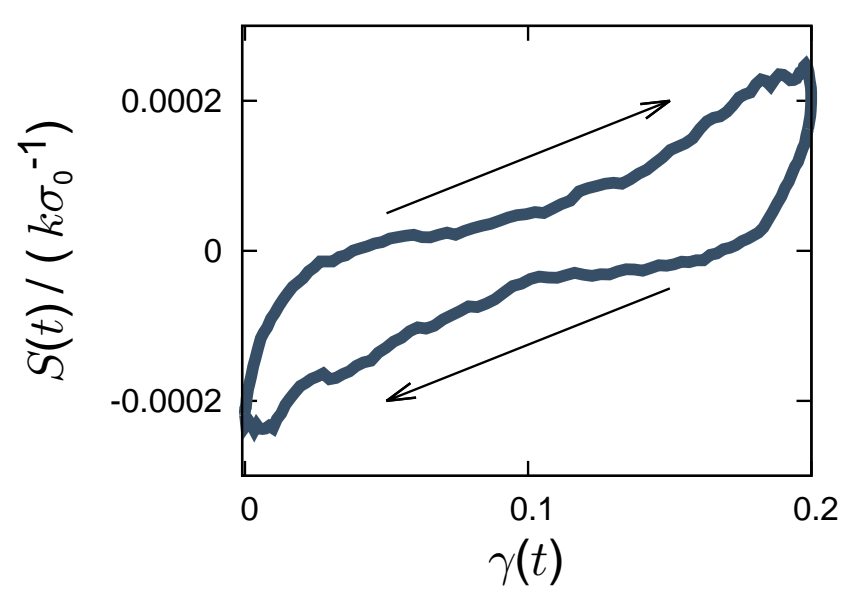

FIG. 2: (Color online) The shear stress $S(t)$ against $\gamma(t)$ with $\phi=0.652$ and $\gamma_{0}=0.1$ for $\Delta=1$.

where $t_{0}\left(\gamma_{0}\right)$ is the time when $\gamma(t)=0$ under the strain amplitude $\gamma_{0}$. Here, the shear stress $S(t)$ is calculated from

$$
\begin{aligned}
S(t)= & -\frac{1}{L^{2}}\left\langle\sum_{i}^{N} \sum_{j>i} r_{i j, x}(t)\left\{f_{i j, y}^{(\mathrm{el})}(t)+f_{i j, y}^{(\mathrm{dis})}(t)\right\}\right\rangle \\
& -\frac{1}{L^{2}}\left\langle\sum_{i=1}^{N} \frac{p_{x, i}(t) p_{y, i}(t)}{2 m}\right\rangle
\end{aligned}
$$

In this paper, we do not analyze the loss modulus because i) it has only the linear dependence on $\omega$ in our simulation and ii) it seems to be independent of density. Note that the stress $S(t)$ exhibits a strong nonlinearity on the strain $\gamma(t)$ as shown in Fig. 2, where we plot the shear stress $S(t)$ against $\gamma(t)$ with $\phi=0.652$ and $\gamma_{0}=0.1$ for $\Delta=1$. It should also be noted that $G$ is almost independent of $\omega$ for $\gamma_{0} \leq 10^{-2}$. We, thus, investigate only $\gamma_{0}$ and $\phi$ dependence of $G$ in this paper.

\section{NUMERICAL RESULTS}

In Fig. 3, we plot $G$ against $\phi-\phi_{J}$ with $\gamma_{0}=$ $10^{-5}, 10^{-3}$ and $10^{-2}$ for $\Delta=1$. It should be noted that the jamming point $\phi_{J}$ is numerically estimated as 0.6494 by the method explained in Appendix A. For the smallest strain amplitude $\left(\gamma_{0}=10^{-5}\right), G$ reproduces the well known behavior Eq. (10 [6, 32], but $G$ seems to satisfy Eq. (2) for large $\gamma_{0}=10^{-2}$. Thus, it is natural to postulate the scaling form Eq. (2) to interpolate two equations.

Figure 4 shows the scaling plot based on Eq. (3) for $\Delta=1$. This figure supports the scaling ansatz, Eq. (3), where we have used exponents

$$
a=0.50 \pm 0.02, \quad b=0.98 \pm 0.02 .
$$

The exponents are determined by the LevenbergMarquardt algorithm [44], where we use the functional

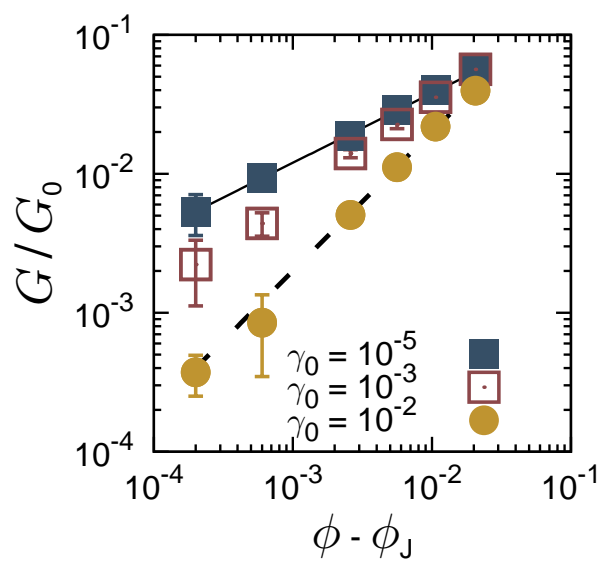

FIG. 3: (Color online) The shear modulus $G$ against the excess volume fraction $\phi-\phi_{J}$ with $\gamma_{0}=10^{-5}, 10^{-3}$, and $10^{-2}$ for $\Delta=1$. The solid and the dashed lines represent the power law functions with the exponent $1 / 2$ and 1 , respectively.

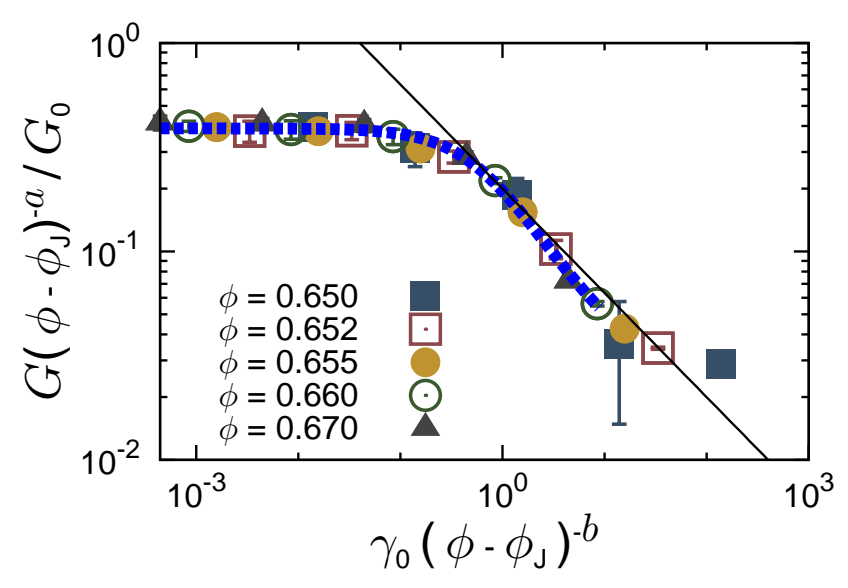

FIG. 4: (Color online) Scaling plot of $G$ characterized by Eq. (3) with $\phi=0.650,0.652,0.655,0.660,0.670$ and $\gamma_{0}=$ $10^{-4}, 10^{-3}, 10^{-2}, 10^{-1}$ for $\Delta=1$. The dashed line is the scaling function given by Eq. (14). The solid line represents the second equation in Eq. (4) with the exponent $c=1 / 2$.

form for the scaling function:

$$
\mathcal{G}(x)=\frac{B_{0}}{1+e^{\sum_{n=1}^{3} B_{n}(\log x)^{n}}}
$$

with fitting parameters $B_{0}=0.39 \pm 0.03, B_{1}=1.1 \pm 0.06$, $B_{2}=-0.08 \pm 0.04$, and $B_{3}=-0.008 \pm 0.008$. Here, we use the critical fraction estimated from a finite size scaling in Appendix A. It should be noted that the estimated values of the exponents do not change within the error margin if we use $\phi_{J}$ as a free parameter in the LevenbergMarquardt algorithm. From Fig. 4, the estimated exponent $c$ in Eq. (44) is approximately given by $1 / 2$. From Eq. (13) and $c=1 / 2$, we obtain $a+b c=0.99 \pm 0.02$, which also supports Eq. (2).

Figure 5 confirms the validity of Eq. (3) for $\Delta=3 / 2$, 


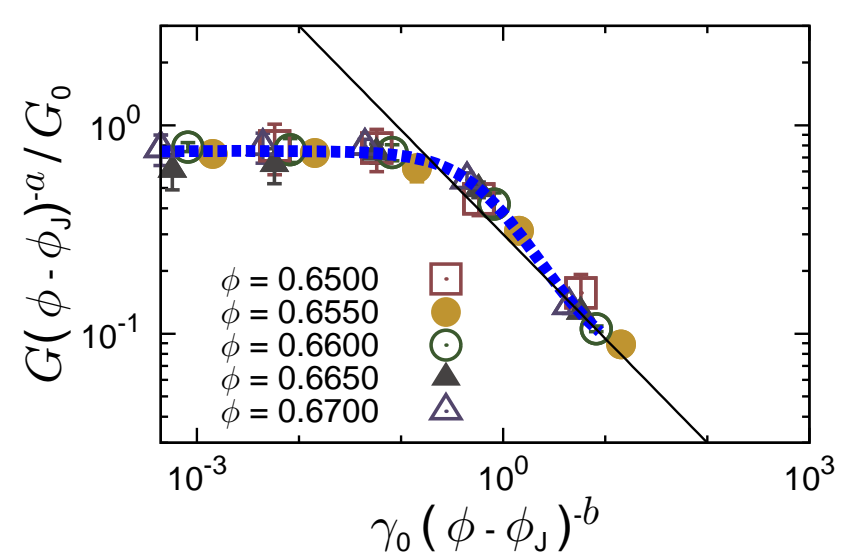

FIG. 5: (Color online) Scaling plot of $G$ characterized by Eq. (3) with $\gamma_{0}=10^{-5}, 10^{-4}, 10^{-3}, 10^{-2}$ and $\gamma_{0}=$ $10^{-4}, 10^{-3}, 10^{-2}, 10^{-1}$ for $\Delta=3 / 2$. The dashed line is the scaling function given by Eq. (14). The solid line represents the second equation in Eq. (4) with the exponent $c=1 / 2$.

where the scaling exponents are numerically estimated as

$$
a=0.99 \pm 0.02, \quad b=0.98 \pm 0.01
$$

with the fitting parameters $B_{0}=0.76 \pm 0.16, B_{1}=$ $1.1 \pm 0.18, B_{2}=-0.089 \pm 0.073, B_{3}=-0.020 \pm 0.016$ and the critical fraction $\phi_{J}=0.6486 \pm 0.0001$, which is numerically estimated by the method explained in Appendix $\mathrm{A}$ The exponent $c$ in Eq. (4) is approximately given by $1 / 2$. It should be noted that the estimated values of the exponents do not change within the error margin if we use $\phi_{J}$ as a free parameter.

\section{PHENOMENOLOGICAL EXPLANATION}

In this section, we try to evaluate the exponents for the scaling law Eqs. (3) and (4) in terms of a mean-field like phenomenological argument. In the first part, we derive the exponent $a$ in Eq. (3). In the second part, we determine the exponent $c$ in Eq. (4). Finally, we discuss the exponent $b$ in Eq. (3).

\section{A. Exponents $a$}

Let us derive the exponent $a$. The relationship between the mean-field compress force $f$ and the compression $\delta$, $f \sim k_{\text {eff }} \delta \sim \delta^{\Delta}$ with the effective spring constant $k_{\text {eff }}$, we immediately obtain the relation $k_{\text {eff }} \sim \delta^{\Delta-1}$. In the vicinity of the jamming point, the compression $\delta$ should satisfy $\delta \sim \phi-\phi_{J}[14]$. On the other hand, from Refs. 32, 45], we may deduce $G / k_{\text {eff }} \sim \delta z \sim \sqrt{\phi-\phi_{J}}$, where $\delta z$ is the excess coordination number and we have used the well known relation: $\delta z \sim \sqrt{\phi-\phi_{J}}$ []]. Thanks to the above relations, we reach $G \sim\left(\phi-\phi_{J}\right)^{\Delta-1 / 2}$. Therefore, we obtain the exponent $a$ as

$$
a=\Delta-1 / 2 .
$$

Equation (16) is consistent with the numerical estimation given by Eqs. (13) and (15) for $\Delta=1$ and $3 / 2$, respectively.

\section{B. Exponent $c$}

We assume that the shear stress under the oscillatory shear is described by a generalized elastic-plastic model [46]. Here, the elastic-plastic model consists of infinite number of series connections with an elastic element of equal shear modulus $G_{0}$ and a slip element characterized by the stress drop $s$ in each avalanche process. We assume that the time evolution of the shear stress $S(t)$ is given by

$$
S(t)=\int_{0}^{\infty} \rho(s) \tilde{S}(s, t) d s,
$$

where $\tilde{S}(s, t)$ is the stress of an individual element having the stress drop $s$, and $\rho(s)$ is the probability density of the stress drop.

We assume that the individual stress $\tilde{S}(s, t)$ for $0 \leq$ $t \leq 2 \pi / \omega$ behaves as a linear function of the strain $\gamma(\bar{t})$ given by Eq. (10) until $|\tilde{S}(s, t)|$ reaches the maximum value $s$, while it drops to 0 when $|\tilde{S}(s, t)|$ exceeds $s$ due to the breakdown of the contact network. Thus, $\tilde{S}(s, t)$ satisfies

$$
\tilde{S}(s, t)= \begin{cases}G_{0} \gamma(t) & \left(0 \leq \theta(t)<\theta_{c}\right) \\ 0 & \left(\theta_{c} \leq \theta(t)<\pi\right) \\ G_{0}\left(\gamma(t)-2 \gamma_{0}\right) & \left(\pi \leq \theta(t)<\pi+\theta_{c}\right) \\ 0 & \left(\pi+\theta_{c} \leq \theta(t)<2 \pi\right)\end{cases}
$$

as illustrated in Fig. 6, where $\theta(t)$ is the phase of the shear strain:

$$
\theta(t)=\omega t .
$$

The explicit expression of the critical phase $\theta_{c}$ for $S(s, t)=s$ is given by

$$
\theta_{c}\left(s /\left(G_{0} \gamma_{0}\right)\right)=\cos ^{-1}\left(1-\frac{s}{G_{0} \gamma_{0}}\right),
$$

where we have used Eqs. (10), (18), and (19).

The expression of the stress-strain relation (17) depends on the probability density $\rho(s)$, which is predicted to satisfy

$$
\rho(s)=A(\phi) s^{-3 / 2} e^{-s / s_{c}(\phi)}
$$

for $s_{0}(\phi) \leq s$, where $s_{0}(\phi)$ and $s_{\mathrm{c}}(\phi)$ are the lower cutoff and the characteristic stress drop, respectively 36 38]. (The derivation and the numerical result for $\rho(s)$ 


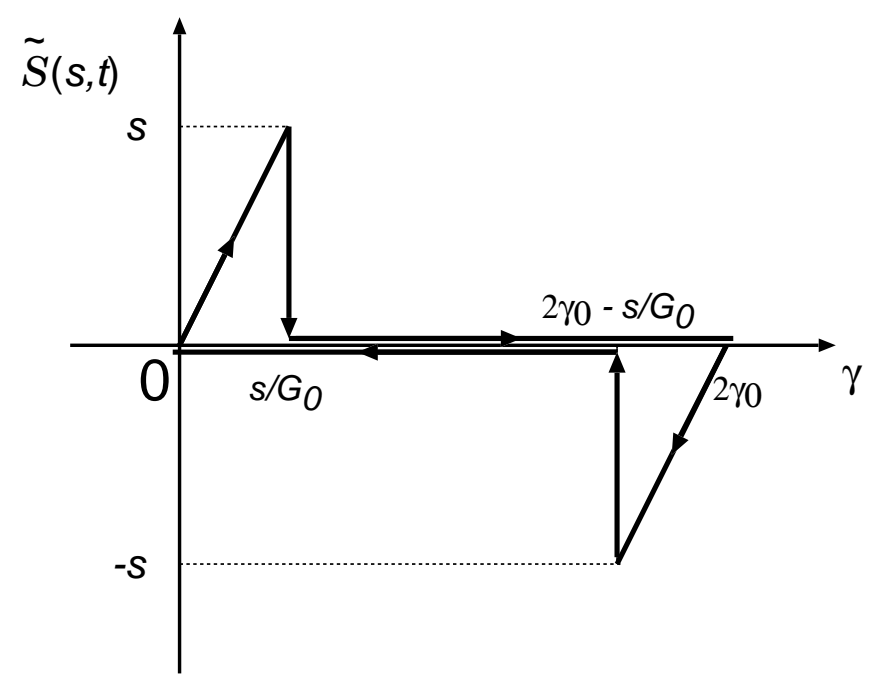

FIG. 6: The stress $\tilde{S}(s, t)$ of an individual element for $0 \leq$ $t \leq 2 \pi / \omega$.

are presented in Appendix B] It should be noted that the cutoff size of the stress drop distribution should exist, because the rearrangement of one grain gives the minimum size of stress drop, though the cutoff might differ from $s_{0}(\phi)$. Here, we simply assume that the distribution lower than $s_{0}(\phi)$ does not contribute to the shear modulus. Here, $A(\phi)$ is the normalization constant satisfying $A(\phi)=1 / \int_{s_{0}(\phi)}^{\infty} d s s^{-3 / 2} e^{-s / s_{c}(\phi)}$, which depends on the volume fraction.

Substituting Eq. (17) into Eq. (11), we obtain

$$
G=\int_{0}^{\infty} d s \tilde{G}\left(\gamma_{0}, s\right) \rho(s)
$$

where $\tilde{G}\left(\gamma_{0}, s\right)$ is the shear modulus of the individual element:

$$
\tilde{G}\left(\gamma_{0}, s\right)=-\frac{\omega}{\pi} \int_{0}^{2 \pi / \omega} d t \frac{\tilde{S}(s, t) \cos (\omega t)}{\gamma_{0}} .
$$

Substituting Eq. (18) into Eq. (23), we obtain

$$
\tilde{G}\left(\gamma_{0}, s\right)=G_{0} F\left(\frac{s}{G_{0} \gamma_{0}}\right)
$$

where

$$
F(x)= \begin{cases}1 & (x \geq 1) \\ T(x) / \pi & (x<1)\end{cases}
$$

with

$$
T(x)=\theta_{c}(x)-2 \sin \theta_{c}(x)+\frac{\sin 2 \theta_{c}(x)}{2} .
$$

Substituting Eqs. (21) and (24) into Eq. (22), we obtain

$$
G=A(\phi) G_{0} \int_{s_{0}}^{\infty} d s s^{-3 / 2} e^{-s / s_{\mathrm{c}}(\phi)} F\left(\frac{s}{G_{0} \gamma_{0}}\right) .
$$

Using $x=s / G_{0} \gamma_{0}$ and expansion $e^{-x\left(G_{0} \gamma_{0}\right) / s_{\mathrm{c}}}=1-$ $x\left(G_{0} \gamma_{0}\right) / s_{\mathrm{c}}+\cdots$ for $s \gg s_{\mathrm{c}}(\phi)$, we obtain

$$
\begin{aligned}
G= & A(\phi) G_{0}^{1 / 2} \gamma_{0}^{-1 / 2} \\
& \times\left\{\int_{\frac{s_{0}}{G_{0} \gamma_{0}}}^{\infty} d x x^{-3 / 2} F(x)+O\left(\frac{G_{0} \gamma_{0}}{s_{\mathrm{c}}}\right)\right\}
\end{aligned}
$$

For $s_{0} / G_{0} \ll \gamma_{0} \ll s_{\mathrm{c}} / G_{0}$, the second term in this equation is negligible and the lower cutoff of the integral can be $s_{0} /\left(G_{0} \gamma_{0}\right) \rightarrow 0$. Then, $G$ is approximately given by

$$
G \simeq A(\phi) G_{0}^{1 / 2} \gamma_{0}^{-1 / 2} \int_{0}^{\infty} d x x^{-3 / 2} F(x) .
$$

Because the integral in Eq. (29) is apparently converged, we obtain

$$
c=1 / 2
$$

from Eqs. (3) and (4).

\section{Exponents $b$}

From Eqs. (16) and (30) with the aid of Eqs. (3) and (44), Eq. (2) for the general $\Delta$ is replaced by

$$
G \sim \gamma_{0}^{-1 / 2}\left(\phi-\phi_{J}\right)^{\Delta+(b-1) / 2}
$$

It should be noted that the dimensions of the shear modulus $G$ and the pressure $P$ are identical, $G$ under large strain amplitude $\gamma_{0}$ might obey the same dependence on $\left(\phi-\phi_{J}\right)$ as that of $P[6], P \sim\left(\phi-\phi_{J}\right)^{\Delta}$, which leads to $b=1$. This is consistent with the numerical estimation given by Eqs. (13) and (15) for $\Delta=1$ and $3 / 2$, respectively. Thus, we believe that $b=1$ can be used in our setup, which is consistent with the resect experiments [47].

\section{DISCUSSION AND CONCLUSION}

This section consists of two parts. In the first part, we discuss our results, and we conclude our work in the second part.

\section{A. Discussion}

Now, let us discuss our results. First, we discuss the relationship between our result and the scaling law of $G$ proposed in Ref. [33]. Second, we compare our results with those on the power spectrum of the shear stress. Finally, we mention the effect of the friction on the scaling for the shear modulus.

Tighe reported that the shear modulus $G$ satisfies a power law of the angular frequency $\omega$ for the oscillatory shear at the jamming point:

$$
G \sim \omega^{1 / 2}
$$


for an analysis of a model of emulsions [33]. In contrast, both our simulation and phenomenology suggest that the shear modulus is independent of $\omega$. We believe that his viscous force preventing grains from the rotation and the sliding is the origin of the nontrivial relation (32) [45], which is not involved in our model in Eq. (6). This is the reason for the absence of the $\omega$-dependence of $G$ in our results.

In a simulation and an experiment of granular materials under steady shear 37, 48], the power spectrum of the shear stress exhibits a non-trivial power law dependence on the frequency $\omega$. In contrast, such a dependence of $G$ does not exist in our simulation under oscillatory shear. It should be noted that the power spectrum is directly related to the time correlation of the stress, but the shear modulus $G$ is related to the average of the stress, which is the origin of the different $\omega$-dependences. To study power spectrum of the shear stress would be one of our future subjects.

It is known that the rheology is drastically affected by friction between particles, at least, for assemblies of soft grains under steady shear [23, 25 29]. The friction plays a key role to cause the shear thickening in rheology, and thus, study on the rheology of frictional grains under an oscillatory shear is practically important. The friction dependence of the scaling law (3) will be discussed elsewhere.

\section{B. Summary}

In conclusion, we numerically study the frictionless granular particles and propose a new scaling law which interpolate between $G \sim\left(\phi-\phi_{J}\right)^{\Delta-1 / 2}$ for infinitesimal strain and $G \sim \gamma_{0}^{-1 / 2}\left(\phi-\phi_{J}\right)^{\Delta}$ for finite strain, where $\Delta$ is the exponent to characterize the local elastic interaction between contacting grains. These scaling exponents are verified through our simulation. The scaling of the shear strain under the large strain can be understood by the theory of slip avalanches.

\section{Acknowledgments}

The authors thanks B. P. Tighe, K. Kamrin, H. Yoshino, K. Miyazaki, S. Titel, and T. Yamaguchi for fruitful discussions, and K. Saitoh and K. Suzuki for their critical reading of the manuscript. The authors also wish to thank Aspen Center for Physics, where parts of this work is developed. This work was supported by JSPS KAKENHI (Grant Nos. 25287098, 22540398, and 25800220) and in part by the Yukawa International Program for Quark-Hadron Sciences (YIPQS). One of the authors (MO) appreciates the warm hospitality of Yukawa Institute for Theoretical Physics at Kyoto University and the discussions during the YITP workshop YITP-W-13-04 on "Physics of glassy and granular materials", YITP-T-13- 03 on "Physics of Granular Flow", and YITP-W-10-20 on "Recent Progress in Physics of Dissipative Particles" to complete this work.

\section{Appendix A: Determination of transition point}

In this appendix, we explain how to determine the critical volume fraction $\phi_{J}$. Here, we assume that $\phi_{J}$ is the volume fraction where the pressure $P$ in the system of $N \rightarrow \infty$ becomes finite under sufficiently small and slow shear strain. We, thus, introduce $f$ as the fraction of samples where $P$ is larger than a threshold value $P_{\mathrm{th}}=10^{-6} k d_{0}^{\Delta-2}$ for $\gamma_{0}=10^{-4}$ and $\omega \tau=10^{-4}$. It should be noted that the estimated $\phi_{J}$ is independent of the choice of $P_{\text {th }}$ within the error margin, at least, for $5.0 \times 10^{-7}<P_{\mathrm{th}} /\left(k d_{0}^{\Delta-2}\right)<1.0 \times 10^{-5}$. Here, $P$ is given by

$$
\begin{aligned}
P= & \frac{1}{3 L^{2}}\left\langle\sum_{i}^{N} \sum_{j>i} \boldsymbol{r}_{i j} \cdot\left(\boldsymbol{f}_{i j}^{(\mathrm{el})}+\boldsymbol{f}_{i j}^{(\mathrm{dis})}\right)\right\rangle \\
& +\frac{1}{3 L^{2}}\left\langle\sum_{i=1}^{N} \frac{\left|\boldsymbol{p}_{i}\right|^{2}}{2 m}\right\rangle .
\end{aligned}
$$

Figures 7 and 8 plot the jammed fraction $f$ against $\phi$ for $\Delta=1$ and $3 / 2$, respectively. Here, $f$ is zero for low $\phi$ and $f$ is finite for large $\phi$. It should be noted that the slope of $f$ around $\phi=0.65$ becomes steeper as the system size increases.

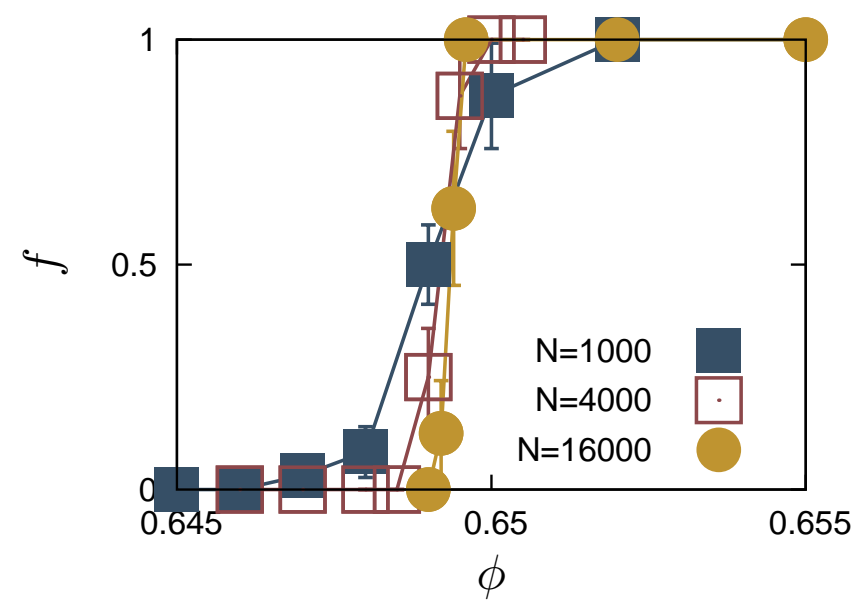

FIG. 7: (Color online) The jammed fraction $f$ against $\phi$ with different system sizes $N=1000,4000$, and 16000 for $\Delta=1$.

In order to determine $\phi_{J}$ from the data in Figs. 7 and 8. we assume $f(\phi, N)$ satisfies a scaling relation

$$
f(\phi, N)=H\left(\left(\phi-\phi_{J}\right) N^{\alpha}\right)
$$

with an exponent $\alpha$ and a scaling function $H(x)$ which satisfies $\lim _{x \rightarrow \infty} H(x)=1$ and $\lim _{x \rightarrow-\infty} H(x)=0$. Figures 9 and 10 verify the assumption (A2), and thus, we can determine $\phi_{J}=0.6494 \pm 0.0001$ and $\phi_{J}=$ 


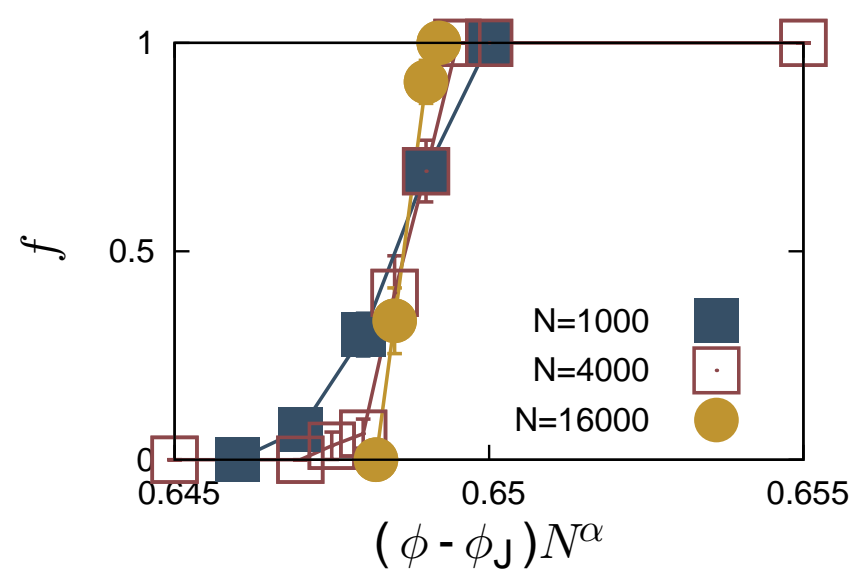

FIG. 8: (Color online) The jammed fraction $f$ against $\phi$ with different system sizes $N=1000,4000$, and 16000 for $\Delta=3 / 2$.

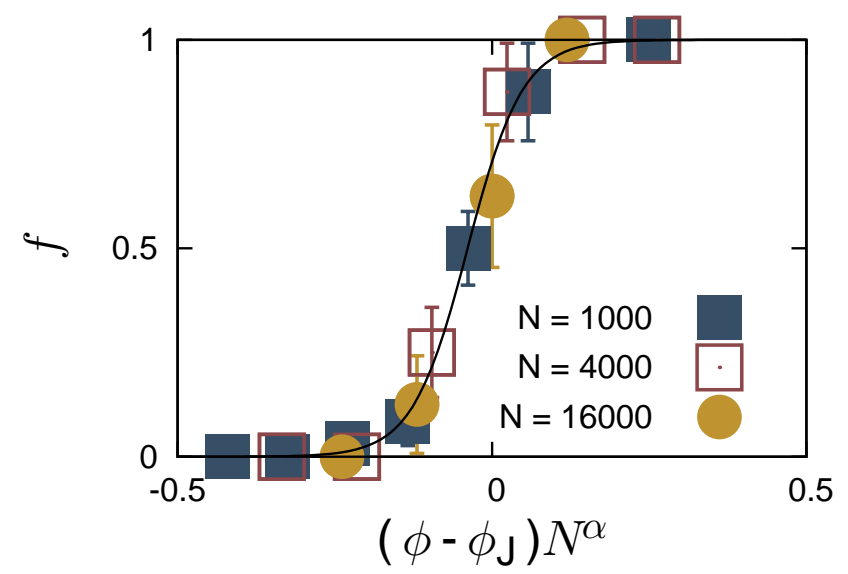

FIG. 9: (Color online) Scaling plot of the jammed fraction $f$ characterized by Eq. (A2) for $\Delta=1$. The solid line is the scaling function given by Eq. A3.

$0.6486 \pm 0.0001$, respectively. Here, we have assumed the functional form of the scaling function as

$$
H(x)=\left\{1+\tanh \left(A_{0}+A_{1} x\right)\right\} / 2
$$

with the fitting parameters $A_{0}=0.4 \pm 0.2, A_{1}=11 \pm 6$ and $\alpha=0.66 \pm 0.07$ for $\Delta=1$, while $A_{0}=0.06 \pm 0.1, A_{1}=$ $45 \pm 41$ and $\alpha=0.42 \pm 0.12$ for $\Delta=3 / 2$.

It should be noted that we estimate $\phi_{J}$ from $P$ at the state with $\gamma_{0}=10^{-4}$, which is obtained by varying the strain amplitude from $10^{0}$ to sequentially decreasing values as $\gamma_{0}=10^{0}, 10^{-1}, 10^{-3}, 10^{-4}$. The estimated value of $\phi_{J}$ depends on the detail of the protocol to decrease $\gamma_{0}$, which might be the origin of the difference of $\phi_{J}$ for $\Delta=1$ and $3 / 2$.

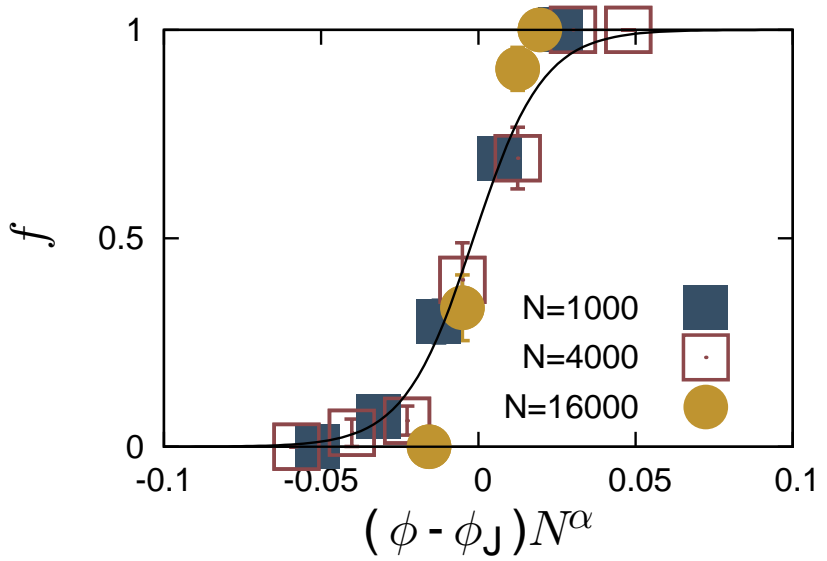

FIG. 10: (Color online) Scaling plot of the jammed fraction $f$ characterized by Eq. (A2) for $\Delta=3 / 2$. The solid line is the scaling function given by Eq. A3.

\section{Appendix B: Distribution of Avalanche size}

In this appendix, we re-derive the probability density $\rho(s)$ of the stress drop $s$ obtained in Refs. [36, 37].

\section{Setup}

In Refs. 36, 37], sheared granular materials are modeled as a simplified lattice system on a coarse-grained scale (larger than the grain diameter) consisting of $N^{\prime}$ sites and the linear size $L$. We apply a strain by moving one boundary at a slow speed $V$ (see Fig. 11).

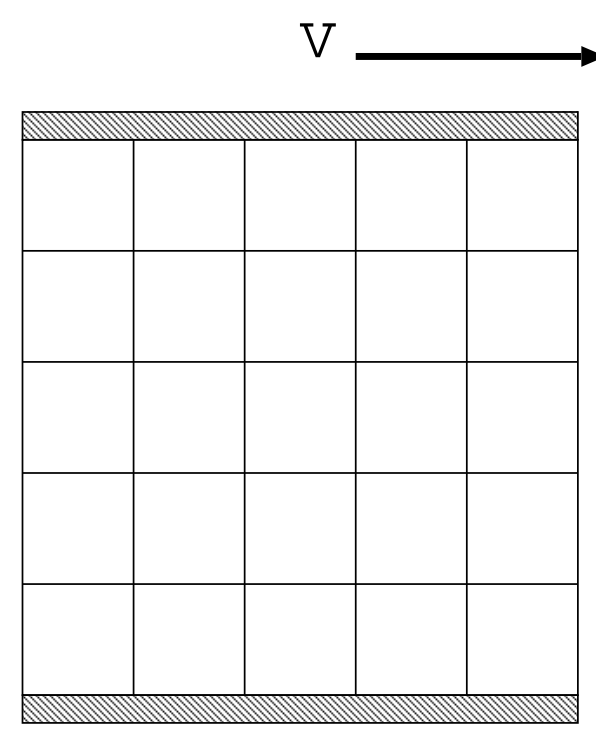

FIG. 11: Illustration of the sheared model lattice.

In this setup, the local shear stress $\sigma_{i}$ at site $i$ under 
the mean field approximation may be given by

$$
\sigma_{i}=K\left(V t-u_{i}\right)+\frac{J}{N^{\prime}} \sum_{j=1}^{N^{\prime}}\left(u_{j}-u_{i}\right),
$$

where $u_{i}$ is the displacement at site $i$. The first term on the right hand side (RHS) of Eq. (B1) represents the contribution of the global shear under the elastic constant $K$, which may satisfy the relation $K \sim G_{0} / L$. The second term on RHS of Eq. (B1) represents the meanfield interaction with the coupling constant $J / N^{\prime}$. We can rewrite Eq. (B1) as

$$
\sigma_{i}=K V t+J \bar{u}-(K+J) u_{i},
$$

where we have introduced

$$
\bar{u}=\sum_{j=1}^{N^{\prime}} u_{j} / N^{\prime} .
$$

The stress $\sigma$ of the system is defined as the average of $\sigma_{i}$ :

$$
\sigma=\frac{1}{N^{\prime}} \sum_{i=1}^{N^{\prime}} \sigma_{i} .
$$

When the local stress $\sigma_{i}$ is lower than the local yield stress $\sigma_{\mathrm{y}}$, we regard the site $i$ as a sticked site, where the displacement $u_{i}$ does not change. As time $t$ goes on, the local stress $\sigma_{i}$ given by Eq. (B2) increases. When the shear stress $\sigma_{i}$ exceeds $\sigma_{\mathrm{y}}$, we assume that the site $i$ slips in the shear direction and $u_{i}$ grows to relax the shear stress $\sigma_{i}$ to the 'arrest stress' $\sigma_{\mathrm{a}}$. The time scale for the local slip may be sufficiently small so that $V t$ in Eq. (B1) is regarded as unchanged during a slip. Thus, the displacement $\delta u_{i}$ and the local stress drop $s_{\text {self }}$ due to the slip are respectively rewritten as

$$
\begin{gathered}
\delta u_{i}=-\frac{\sigma_{\mathrm{y}}-\sigma_{\mathrm{a}}}{K+J}, \\
s_{\text {self }}=-\left(\sigma_{\mathrm{y}}-\sigma_{\mathrm{a}}\right),
\end{gathered}
$$

which leads to the increase of the local stress at the other sites as

$$
s_{\mathrm{oth}}=C\left(\sigma_{\mathrm{y}}-\sigma_{\mathrm{a}}\right) / N^{\prime}
$$

with

$$
C=\frac{J}{J+K}
$$

Then, the stress drop $s$ of the total system is approximately given by $-(1-C)\left(\sigma_{\mathrm{y}}-\sigma_{\mathrm{a}}\right) / N^{\prime}$.

This increase of the local stress may lead to the slip of a site $j \neq i$, and result in a sequential avalanche with $n$ slips, where the stress drop is given by

$$
s=(1-C)\left(\sigma_{\mathrm{y}}-\sigma_{\mathrm{a}}\right) n / N^{\prime} .
$$

\section{Derivation of $\rho(s)$}

As time goes on, the system is expected to reach a statistical steady state. In this subsection, we derive the probability of the stress drop $s$ in the steady state.

Let us consider the distribution of $\sigma_{i}$ just before the avalanche begins in order to derive the probability of $s$. Here, we introduce a variable $X_{n}$ as

$$
X_{n}=\sigma_{i(n+1)},
$$

where $i(n)$ is the index of the site that has the $n$th largest stress (see Fig. 12). The largest value $X_{0}$ is $\sigma_{\mathrm{y}} . X_{n}$ decreases as $n$ increases with the gap

$$
\delta X_{n}=X_{n-1}-X_{n},
$$

which is randomly distributed.

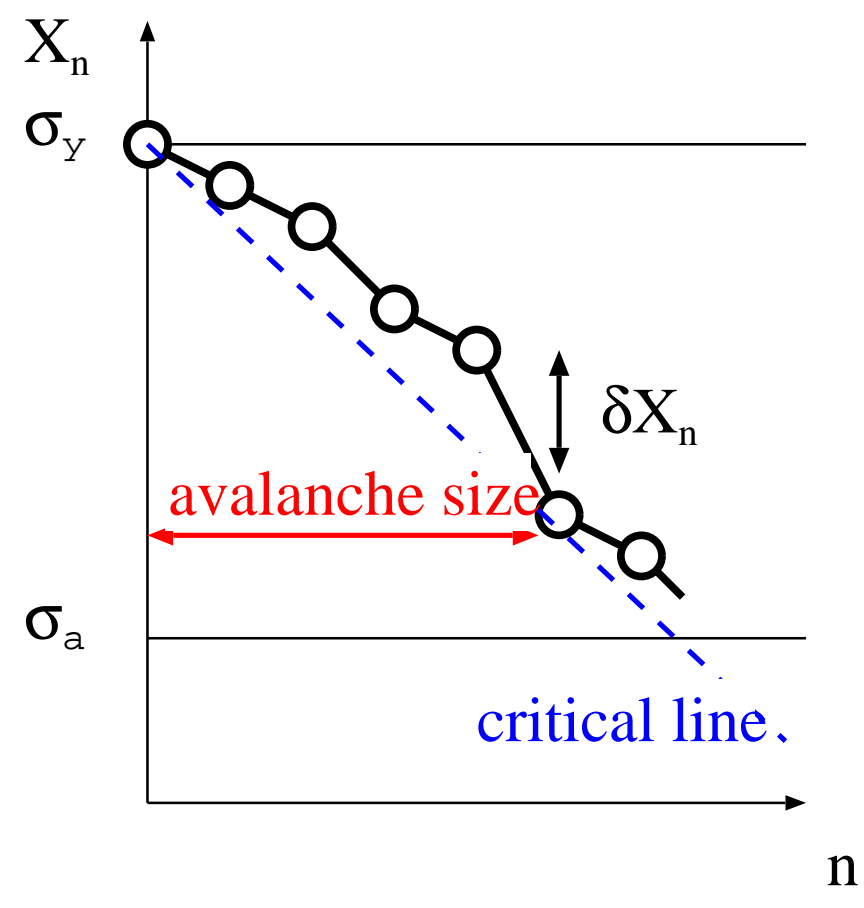

FIG. 12: The schematic picture of $X_{n}$. The solid line is the critical line $\sigma_{\mathrm{y}}-n s_{\text {oth }}$.

Assuming that $\sigma_{i}$ is likely to take any allowable value between $\sigma_{\mathrm{a}}$ and $\sigma_{\mathrm{y}}, X_{n}$ obeys a Poisson process. The probability of the intervals divided by variables obeying a Poisson process satisfies an exponential distribution [49]. Therefore, the distribution of $\delta X_{n}$ is given by

$$
\rho_{X}\left(\delta X_{n}\right)=\frac{N^{\prime}}{\sigma_{\mathrm{y}}-\sigma_{\mathrm{a}}} e^{-\frac{N^{\prime}}{\sigma_{\mathrm{y}}-\sigma_{\mathrm{a}}} \delta X_{n}} .
$$

When the avalanche starts, the site $i(1)$ slips and the local stress at other sites increases by $s_{\text {oth }}=C\left(\sigma_{\mathrm{y}}-\right.$ $\left.\sigma_{\mathrm{a}}\right) / N^{\prime}$. If the local stress at the site $i(2)$ exceeds $\sigma_{\mathrm{y}}$ because of the increase of the stress, it slips. This means 
that the slip proceeds to the site $i(2)$ if $X_{1}$ is larger than $\sigma_{\mathrm{y}}-s_{\mathrm{oth}}$. Similarly, the site $i(n+1)$ slips if $X_{n}$ is larger than $\sigma_{\mathrm{y}}-n s_{\text {oth. }}$. In Fig. 12, we plot the critical line $\sigma_{\mathrm{y}}-n s_{\text {oth }}$. Therefore, the size of the avalanche of the sample shown in this figure is given by the length of the region where $X_{n}$ exceeds the critical line.

In order to obtain the probability distribution of the avalanche size, we define

$$
Z_{n}=X_{n}-\left(\sigma_{\mathrm{y}}-n s_{\mathrm{oth}}\right) .
$$

We plot the schematic illustration of $Z_{n}$ in Fig. 13, The avalanche size is the length of the region where $Z_{n}$ exceeds 0 . Since $Z_{n}$ is considered as a biased random walk, the avalanche size is calculated as the first passage time of the biased random walk.

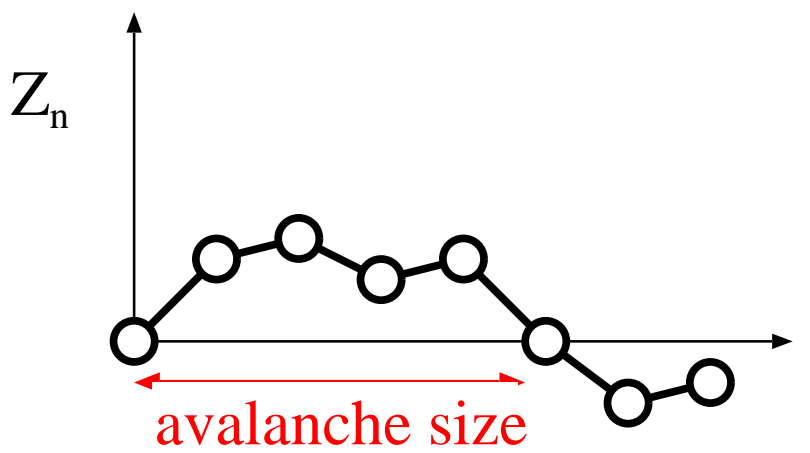

FIG. 13: The schematic illustration of $Z_{n}$.

Here, we assume that $\delta Z_{n}=Z_{n}-Z_{n-1}$ obeys a Bernoulli trial which has $\Delta x$ and $-\Delta x$ with the probability $p$ and $1-p$, respectively. Then, the average $\mu_{Z}$ and the variance $V_{Z}$ are given by

$$
\begin{gathered}
\mu_{Z}=(2 p-1) \Delta x, \\
V_{Z}=4 \Delta x^{2} p(1-p) .
\end{gathered}
$$

Because $\delta Z_{n}=Z_{n}-Z_{n-1}$ is rewritten with Eq. (B13) as

$$
\delta Z_{n}=-\delta X_{n}+s_{\text {oth }}
$$

and the probability distribution of $\delta X_{n}$ satisfies Eq. (B12), $\mu_{Z}$ and $\sigma_{Z}$ are respectively given by

$$
\begin{gathered}
\mu_{Z}=-(1-C) \frac{\sigma_{\mathrm{y}}-\sigma_{\mathrm{a}}}{N^{\prime}}, \\
V_{Z}=\frac{\left(\sigma_{\mathrm{y}}-\sigma_{\mathrm{a}}\right)^{2}}{N^{\prime 2}} .
\end{gathered}
$$

From Eqs. (B14), (B15), (B17), and (B18), the probability $p$ and the step size $\Delta x$ are respectively given by

$$
\begin{aligned}
p & =\frac{1}{2}\left(1+\frac{C-1}{\sqrt{1+(C-1)^{2}}}\right), \\
\Delta x & =\sqrt{1+(C-1)^{2}} \frac{\sigma_{\mathrm{y}}-\sigma_{\mathrm{a}}}{N^{\prime}} .
\end{aligned}
$$

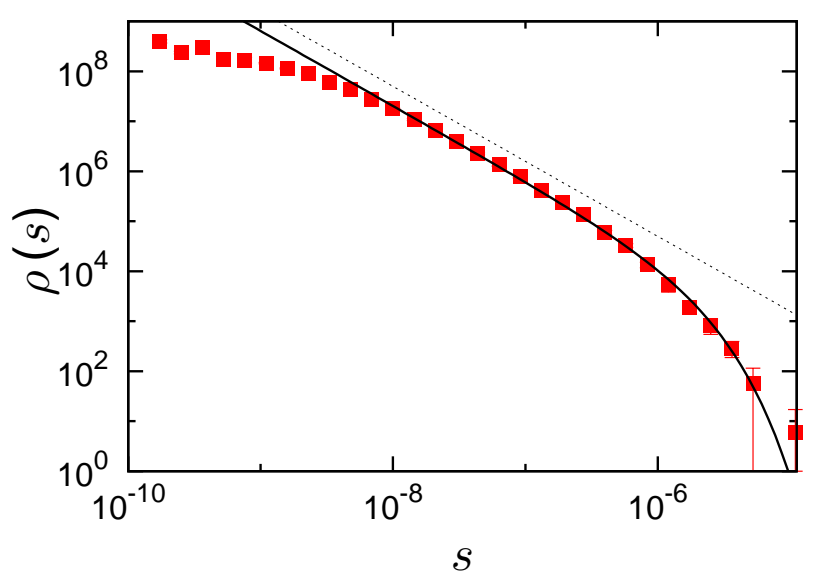

FIG. 14: (Color online) The probability density $\rho(s)$ against $s$ for $\phi_{J}=0.6700, \gamma_{0}=10^{-2}$ with $\Delta=1$. The dotted and solid lines, respectively, represent the power law function with the exponent $3 / 2$ and Eq. (21) with $A=2.0 \times 10^{-5}, s_{\mathrm{c}}=$ $1.4 \times 10^{-6}$.

Here, we introduce $\lambda_{n}$ as the probability that $Z_{n}=$ $\sum_{m=1}^{n} \delta Z_{m}$ becomes negative for the first time at the $n$ th step. As shown in Ref. [49], such probability for the first passage problem is given by

$$
\begin{aligned}
\lambda_{2 n-1} & =0 \\
\lambda_{2 n} & =\frac{1}{2 p}\left(\begin{array}{c}
1 / 2 \\
n
\end{array}\right)(-1)^{(n+1)}\{4 p(1-p)\}^{n} .
\end{aligned}
$$

With the aid of Stirling's formula with Eq. (B20), $\lambda_{2 n}$ for sufficiently large $n$ is approximately given by

$$
\lambda_{2 n}=\frac{1}{4 \pi^{1 / 2} p} \frac{1}{n^{3 / 2}} e^{-n / n_{c}}
$$

with $n_{c}=-1 / \log (4 p(1-p))=1 / \log \left(1+(C-1)^{2}\right)$.

Because the avalanche size $n$ is proportional to the stress drop $s$ as shown in (B9), the probability density $\rho(s)$ of the stress drop $s$ is thus approximately given by Eq. (21).

Figure 14 is the numerical result of the stress drop, which well reproduces Eq. (21) in the region $s>10^{-8}$, where the probability density $\rho(s)$ against $s$ for $\phi_{J}=$ $0.6700, \gamma_{0}=10^{-2}$ with $\Delta=1$ is shown.
[1] H. M. Jaeger, S. R. Nagel, and R. P. Behringer, Rev. Mod. Phys. 68 (1996), 1259.
[2] P. N. Pusey, in Liquids, Freezing and the Glass Transi- 
tion, Part II Les Houches Summer School Proceedings Vol. 51, edited by J. -P. Hansen, D. Levesque, and J. Zinn-Justin (Elsevier, Amsterdam, 1991), Chap. 10.

[3] D. J. Durian and D. A. Weitz, "Foams," in Kirk-Othmer Encyclopedia of Chemical Technology, 4th ed., edited by J. I. Kroschwitz (Wiley, New York, 1994), Vol. 11, p. 783.

[4] A. J. Liu and S. R. Nagel, Nature 396 (1998), 21.

[5] C. S. O'Hern, S. A. Langer, A. J. Liu, and S. R. Nagel, Phys. Rev. Lett. 88, 075507 (2002).

[6] C. S. O'Hern, L. E. Silbert, A. J. Liu, and S. R. Nagel, Phys. Rev. E 68, 011306 (2003).

[7] M. Wyart, L. E. Silbert, S. R. Nagel, and T. A Witten, Phys. Rev. E 72, 051306 (2005).

[8] T. S. Majmudar, M. Sperl, S. Luding, and R. P. Behringer, Phys. Rev. Lett. 98, 058001 (2007).

[9] P. Olsson and S. Teitel, Phys. Rev. Lett. 99, 178001 (2007).

[10] T. Hatano, M. Otsuki, and S. Sasa, J. Phys. Soc. Jpn. 76, 023001 (2007).

[11] T. Hatano, J. Phys. Soc. Jpn. 77, 123002 (2008).

[12] B. P. Tighe, E. Woldhuis, J. J. C. Remmers, W. van Saarloos, and M. van Hecke, Phys. Rev. Lett. 105, 088303 (2010).

[13] T. Hatano, Prog. Theor. Phys. Suppl. 184, 143 (2010).

[14] M. Otsuki and H. Hayakawa, Prog. Theor. Phys. 121, 647 (2009).

[15] M. Otsuki and H. Hayakawa, Phys. Rev. E 80, 011308 (2009).

[16] M. Otsuki, H. Hayakawa, and S. Luding, Prog. Theor. Phys. Suppl. 184, 110 (2010).

[17] K. N. Nordstrom, E. Verneuil, P. E. Arratia, A. Basu, Z. Zhang, A. G. Yodh, J. P. Gollub, and D. J. Durian, Phys. Rev. Lett. 105, 175701 (2010).

[18] P. Olsson and S. Teitel, Phys. Rev. E 83, 030302(R) (2011).

[19] D. Vågberg, P. Olsson, and S. Teitel, Phys. Rev. E 83, 031307 (2011).

[20] M. Otsuki and H. Hayakawa, Prog. Theor. Phys. Suppl. No. 195, 129 (2012).

[21] A. Ikeda, L. Berthier, and P. Sollich, Phys Rev Lett. 109 018301 (2012).

[22] P. Olsson and S. Teitel, Phys. Rev. Lett. 109, 108001 (2012).

[23] M. Otsuki and H. Hayakawa, Phys. Rev. E 83, 051301 (2011).

[24] D. Bi, J. Zhang, B. Chakraborty and R. Behringer, Nature 480, 355 (2011).

[25] S. Chialvo, J. Sun, and S. Sundaresan, Phys. Rev. E 85, 021305 (2012).

[26] E. Brown and H. M. Jaeger, Phys. Rev. Lett. 103, 086001 (2009).

[27] R. Seto, R. Mari, J. F. Morris, and M. M. Denn, Phys. Rev. Lett. 111, 218301 (2013).

[28] N. Fernandez, R. Mani, D. Rinaldi, D. Kadau, M. Mos- quet, H. Lombois-Burger, J. Cayer-Barrioz, H. J. Herrmann, N. D. Spencer, and L. Isa, Phys. Rev. Lett. 111, 108301 (2013).

[29] C. Heussinger, Phys. Rev. E 88, 050201 (2013).

[30] M. M. Bandi, M. K. Rivera, F. Krzakala and R.E. Ecke, Phys. Rev. E 87, 042205 (2013).

[31] M. Wyart and M. Cates, Phys. Rev. Lett. 112, 098302 (2014).

[32] M. Wyart, Annales de Physique 30, 1 (2005).

[33] B. P. Tighe, Phys. Rev. Lett. 107, 158303 (2011).

[34] T. G. Mason, Matin-D. Lacasse, G. S. Grest, D. Levine, J. Bibette, D. A. Weitz, Phys. Rev. E 56, 3150 (1997).

[35] S. Okamura and H. Yoshino, arXiv:1306.2777.

[36] K. Dahmen, D. Ertaş, and Y. Ben-Zion, Phys. Rev. E 58, 1494 (1998).

[37] K. Dahmen, Y. Ben-Zion, and J. T. Uhl, Nat. Phys. 7, 554 (2011).

[38] A. Dobrinevski, PhD thesis, Ecole Normale Sup'erieure (2013), arXiv:1312.7156

[39] M. Wyart, H. Liang, A. Kabla, and L. Mahadevan, Phys. Rev. Lett. 101, 215501 (2008).

[40] M. Otsuki and H. Hayakawa, Powders and Grains 2013: Proceedings of the 7th International Conference on $\mathrm{Mi}$ cromechanics of Granular Media, edited by A. Yu, K. Dong, R. Yang, and S. Luding, AIP Conf. Proc. 1542, 503 (2013).

[41] D. J. Evans and G. P. Morriss, Statistical Mechanics of Nonequilibrium Liquids 2nd ed. (Cambridge University Press, Cambridge, 2008).

[42] In order to obtain the state with $\gamma_{0}$ and $\omega$, we vary the strain amplitude $\gamma_{0}$ from $10^{0}$ to sequentially decreasing values as $\gamma_{0}=10^{0}, 10^{-1}, 10^{-2}, 10^{-3}, 10^{-4}, 10^{-5}$. For each value of $\gamma_{0}$, we sequentially change the values of the frequency $\omega$ as $\omega \tau=10^{-1}, 10^{-2}, 10^{-3}, 10^{-4}$. For a fixed set of $\gamma_{0}$ and $\omega$, we measure the stress in a statistical steady state, where we use the data in the last cycle of the four cycles of the oscillatory shear.

[43] M. Doi and S. F. Edwards, The Theory of Polymer Dynamics (Oxford University Press, Oxford, 1990).

[44] W. H. Press, S. A. Teukolsky, W. T. Vetterling, and B. P. Flannery, Numerical Recipes, 3rd ed. (Cambridge University Press, Cambridge, UK, 2007).

[45] B. Tighe, arXiv:1205.2960

[46] V. A. Lubarda, D. Sumarac, and D. Krajcinovic, Eur. J. Mech., A/Solids 12, 445 (1993).

[47] C. Coulais, A. Seguin, and O. Dauchot, arXiv:1403.5885, to be published in PRL.

[48] F. Dalton and D. Corcoran, Phys. Rev. E 63, 061312 (2001).

[49] W. Feller, An Introduction to Probability Theory and Its Applications, 2nd ed. (John Wiley \& Sons, Inc., New York, 1957). 